\title{
Glue Embolization of a Blunt Traumatic Hepatic Arteriovenous Fistula under Inflow and Outflow Control
}

\author{
Takahiko Mine ${ }^{1}$, Satoru Murata ${ }^{1}$, Daisuke Yasui ${ }^{1}$, \\ Hiroyuki Yokota ${ }^{2}$, Hiroyuki Tajima ${ }^{3}$ and Shin-ichiro Kumita ${ }^{1}$ \\ ${ }^{1}$ Department of Radiology, Nippon Medical School \\ ${ }^{2}$ Department of Emergency and Critical Care Medicine, Nippon Medical School \\ ${ }^{3}$ Center for Minimally Invasive Treatment, Nippon Medical School Musashi Kosugi Hospital
}

\begin{abstract}
We report on a rare case of blunt traumatic hepatic arteriovenous fistula arising from a pseudoaneurysm in a 35-year-old woman. Transarterial embolization was performed with n-butyl-2-cyanoacrylate, under inflow control with loose coil packing within the pseudoaneurysm and outflow control by balloon occlusion of the hepatic vein. A promising therapeutic outcome was achieved without any serious adverse events. Thus, the combination of these techniques to control inflow and outflow was successfully used to treat this rare hepatic vascular injury. (J Nippon Med Sch 2016; 83: 27-30)
\end{abstract}

Key words: blunt liver trauma, hepatic injury, hepatic arteriovenous fistula, embolization, n-butyl-2cyanoacrylate

\section{Introduction}

Traumatic hepatic arteriovenous fistula (AVF) is uncommon; the majority of cases result from penetrating trauma, and extremely few cases are caused by blunt trauma ${ }^{1,2}$. Although transcatheter arterial embolization is now regarded as a key element in the treatment of highgrade hepatic injuries ${ }^{3}$, a technical strategy for rare conditions, such as AVF, has not yet been established. We describe a case in which a hepatic AVF due to blunt trauma successfully underwent endovascular treatment with nbutyl-2-cyanoacrylate (NBCA; B. Braun Melsungen AG, Melsungen, Germany) under inflow control with loose coil packing within the pseudoaneurysm and outflow control by balloon occlusion of the hepatic vein.

\section{Case Presentation}

A 35-year-old woman fell and struck the right upper quadrant of the abdomen on the corner of a table. When she came to us, she had already been treated at another hospital for transient hemorrhagic shock, where hepatic injury had been diagnosed with unenhanced computed tomography $(\mathrm{CT})$. For further examination and treatment she was transferred to the advanced emergency critical care center at our hospital. On arrival, she was conscious and alert, and the vital signs were as follows: systolic blood pressure, $108 \mathrm{~mm} \mathrm{Hg}$; respiratory rate, 21 breaths/ minute; heart rate, 112 beats/min; and oxygen saturation via pulse oximetry, $100 \%$ under $10 \mathrm{~L} \mathrm{O}_{2} /$ minute. The results of laboratory studies were as follows: serum alanine transaminase, $204 \mathrm{U} / \mathrm{I}$; serum aspartate aminotransferase, $746 \mathrm{U} / \mathrm{I}$; and serum total bilirubin $0.4 \mathrm{mg} / \mathrm{dL}$; hemoglobin, $9.4 \mathrm{~g} / \mathrm{dL}$; and hematocrit levels, and $22.0 \%$. The hemodynamic status was not continuously stable; thus, 6 units of packed red blood cells and 6 units of fresh frozen plasma were administered. Contrast-enhanced CT showed a hepatic arterial pseudoaneurysm in the anterior portion of the right hepatic lobe and hemoperitoneum around the liver and spleen (Fig. 1).

Emergency angiography was performed to embolize the pseudoaneurysm. Celiac and selective hepatic angiograms using a 4-French system and 2.0-French microcatheter (Goldcrest Neo; Kosin, Tokyo, Japan) via the femoral artery revealed a pseudoaneurysm at the anterior branches of the right hepatic artery, a high-flow AVF connected to the middle hepatic vein, and arterioportal shunts (Fig. 2a). The AVF arose from the pseudoaneu-

Correspondence to Takahiko Mine, MD, PhD, Department of Radiology, Nippon Medical School, 1-1-5 Sendagi, Bunkyo-ku, To-

kyo 113-8603, Japan

E-mail: s8090@nms.ac.jp

Journal Website (http://www.nms.ac.jp/jnms/) 


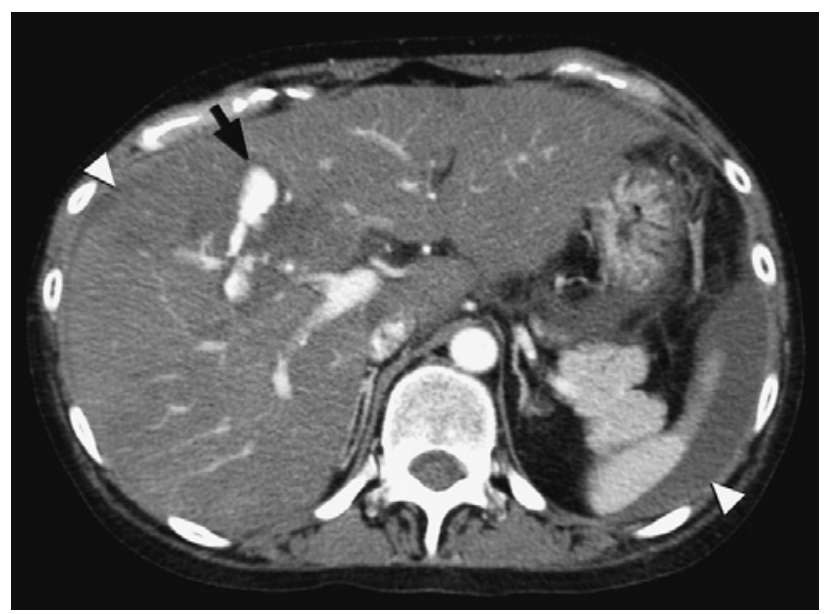

Fig. 1 Contrast-enhanced computed tomography scan before angiography.

The axial image shows a pseudoaneurysm in the anterior portion of the right hepatic lobe (arrow) and hemoperitoneum around the liver and spleen (arrowheads).

rysm, which was located near the branching point of some anterior branches. Because of this complicated anatomical condition, embolization of the arterial side using only coils was considered to be associated with a high risk of technical failure; accordingly, simultaneous embolization of the pseudoaneurysm and AVF using NBCA under inflow and outflow control was instead planned.

First, for outflow control, the middle hepatic vein near the shunt point of the AVF was occluded with a 6-French compliant balloon catheter with a maximum diameter of 20 mm (Selecon; Terumo Clinical Supply Co., Ltd., Gifu, Japan), which was inserted from the right jugular vein (Fig. 2b). The position of the balloon was adjusted to prevent the complete elimination of the venous outflow by preserving the shunt point itself. Second, for inflow control, the neck of the pseudoaneurysm was loosely packed with microcoils (Tornado Embolization Coil; Cook Medical Inc., Bloomington, IN, USA) (Fig. 2c) until the appropriate blood flow reduction was confirmed to preserve a measurable flow to the venous system. Then, 5 coils of 5 -mm diameter and 6 coils of 4 -mm diameter were required for adequate flow control. The NBCA was mixed with iodized oil in a $25 \%$ ratio, and $0.7 \mathrm{~mL}$ of this mixture was administered slowly into the neck of the pseudoaneurysm under this inflow and outflow control, until it was completely filled. Finally, exclusion of the AVF and pseudoaneurysm was confirmed with a hepatic angiogram. Although the arterioportal shunts remained, they were carefully observed.
Four days later, follow-up angiography revealed that the pseudoaneurysm and AVF were cured, and the arterioportal shunts had disappeared (Fig. 2d). No adverse events occurred, and the patient was uneventfully discharged from the hospital 19 days later. Two months later, contrast-enhanced CT showed no recurrence of the pseudoaneurysm or AVF and no serious liver damage (Fig. 3).

\section{Discussion}

Traumatic hepatic AVF caused by blunt trauma is extremely rare, and only 2 previous cases of endovascular treatment for AVFs after penetrating trauma have been reported; 1 case was embolized with an Amplatzer Vascular Plug (St. Jude Medical Inc., St. Paul, MN, USA) and $\mathrm{NBCA}^{1}$, and the other case was embolized with coils ${ }^{2}$. In the present case, we successfully performed embolization with NBCA for a blunt traumatic hepatic AVF under inflow control with loose coil packing within the pseudoaneurysm and outflow control by balloon occlusion of the hepatic vein. To our knowledge, this is the first report of such a case treated with a dual endovascular approach.

In this treatment 2 technical key points were considered. The first was the precise occlusion of the pseudoaneurysm with NBCA. The CT scan showed a pseudoaneurysm in the anterior portion of the right hepatic lobe and hemoperitoneum. These findings indicated active intraparenchymal bleeding and laceration of the liver surface, and the injury was classified as grade IV according to the liver injury grading system guidelines of the American Association for the Surgery of Trauma ${ }^{4}$. Furthermore, the hemodynamic status of our patient could not be stabilized; hence, rapid and complete occlusion of the pseudoaneurysm was essential for survival. Coil embolization of the afferent and efferent arteries is the most appropriate technique for embolization of a ruptured pseudoaneurysm ${ }^{5,6}$; however, when coil embolization fails to control the bleeding, the mortality rate is high, even when subsequent surgery is performed ${ }^{6,7}$. Furthermore, if many adjacent arteries have originated near the pseudoaneurysm, as in our patient, embolizing all of the adjacent arteries using coils may be time-consuming and technically difficult. Therefore, we performed direct embolization of the pseudoaneurysm with NBCA and a coils so that the lesion could be occluded more quickly and securely than with a coil alone. Furthermore, the use of NBCA supports the coil pack and decreases the time required for polymerization ${ }^{8,9}$. Parildar et al. have re- 

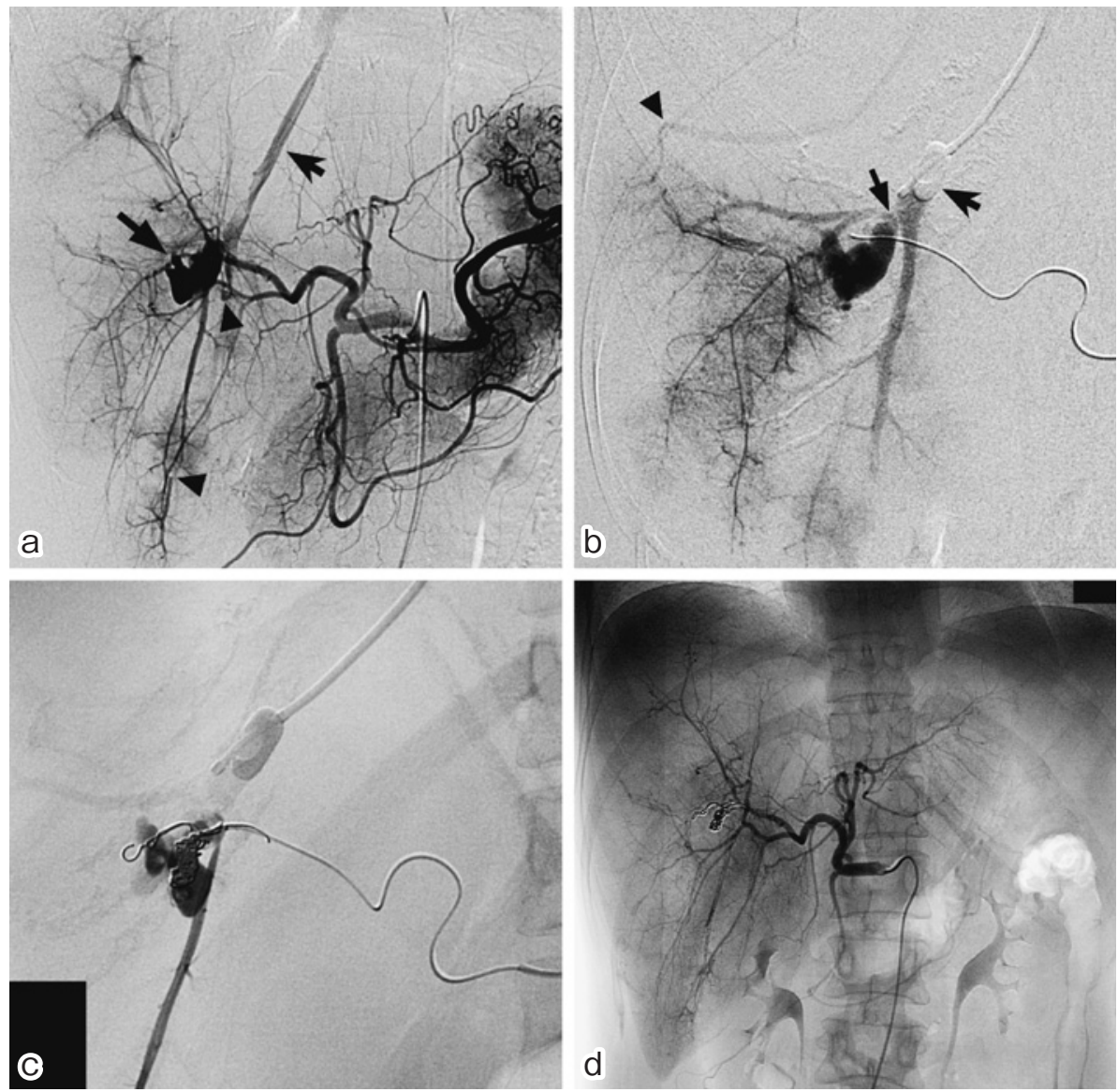

Fig. 2 Emergent angiography and treatment.

a) A celiac angiogram shows a pseudoaneurysm located near the branching point of the anterior branches (arrow), a high-flow hepatic arteriovenous fistula connected to the middle hepatic vein (acute angle arrow), and some arterioportal shunts (arrowheads). b) Selective aneurysmography after occlusion of the middle hepatic vein near the shunt point (arrow) by balloon catheter (acute angle arrow) shows a reduction in venous flow; however, measurable blood flow was retained via the collateral blood flow (arrowhead). c) Aneurysmography after loose packing of the pseudoaneurysm with coils shows preservation of the pseudoaneurysm cavity. d) Follow-up angiography 4 days after treatment shows exclusion of the arteriovenous fistula and pseudoaneurysm and disappearance of the arterioportal shunts.

ported that embolization of a visceral artery aneurysm with NBCA and coils increases the success rate over that of coils alone'. Moreover, the presence of the coil pack reduces blood flow and the risk of NBCA leaking beyond the shunt in cases with arteriovenous shunt disease ${ }^{8}$. In our patient, this advantage was utilized as inflow control.

The second key point considered in this treatment was the adequate delivery of embolization materials for the obliteration of the AVF. When high-flow arteriovenous shunt disease is treated, blood flow control is an extremely important technical factor needed to gain a sufficient embolization effect and to avoid passing materials into the venous system ${ }^{1,10}$. When NBCA is used, complete hemostasis before the injection of NBCA increases the risk of reflux into the normal parenchyma, resulting in tissue infarction. Hence, adjusting the degree of flow reduction is required for adequate flow control ${ }^{10}$. In our patient, we used 2 routes of flow control. For inflow control, the neck of the pseudoaneurysm was loosely packed with microcoils. We carefully preserved the cavity of the pseudoaneurysm and measurable flow, which was confirmed by repeated angiography. For outflow control, the middle hepatic vein near the AVF was occluded by a balloon catheter. To prevent complete elimination of the venous outflow, we did not occlude the shunt point. Through the use of these controls, satisfactory flow reduction was achieved, which preserved a measurable 


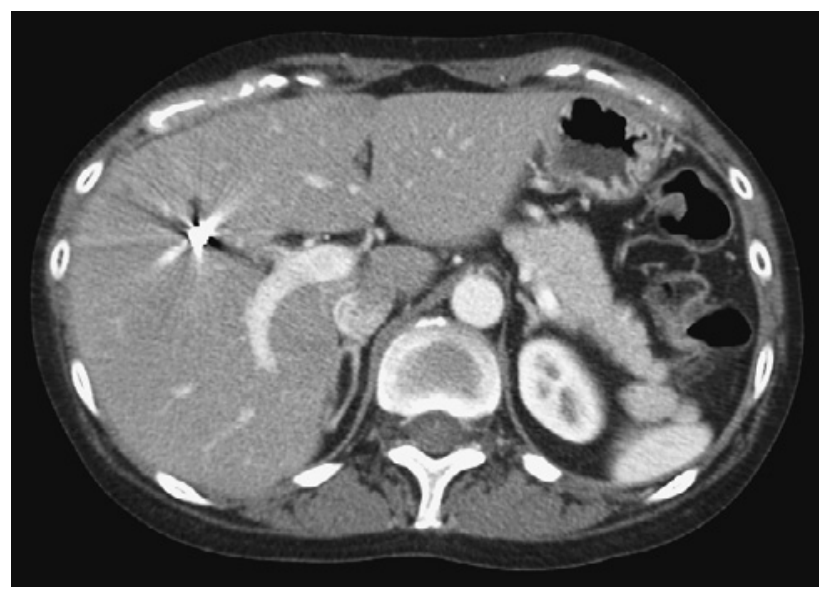

Fig. 3 Follow-up contrast-enhanced computed tomography scan 2 months after treatment.

Computed tomography shows no recurrence of the pseudoaneurysm or arteriovenous fistula and no serious liver damage.

blood flow to the venous system. Finally, NBCA was administered safely and without reflux, and the AVF and pseudoaneurysm were completely excluded.

In conclusion, glue embolization using a dual approach with inflow and outflow control was successfully used to treat blunt traumatic hepatic AVF. On the basis of this case, we recommend this approach as a radical option for treating these rare hepatic vascular injuries.

Conflict of Interest: The authors declare that they have no competing interests.

\section{References}

1. Koc O, Cil BE, Peynircioglu B, Emlik D, Ozbek O: Com- plementary use of NBCA with the Amplatzer vascular plug for embolization of a high-flow traumatic hepatic arteriovenous fistula. Cardiovasc Intervent Radiol 2009; 32: 1105-1107.

2. Mayo A, Aladgem D, Makrin V, Kluger Y: Traumatic hepatic artery pseudo-aneurysm with fistula to the hepatic vein. Isr Med Assoc J 2004; 6: 496-497.

3. Letoublon C, Morra I, Chen Y, Monnin V, Voirin D, Arvieux C: Hepatic arterial embolization in the management of blunt hepatic trauma: indications and complications. J Trauma 2011; 70: 1032-1037.

4. Yoon W, Jeong YY, Kim JK, Seo JJ, Lim HS, Shin SS, Kim JC, Jeong SW, Park JG, Kang HK: CT in blunt liver trauma. Radiographics 2005; 25: 87-104.

5. Reber PU, Baer HU, Patel AG, Wildi S, Triller J, Büchler MW: Superselective microcoil embolization: treatment of choice in high-risk patients with extrahepatic pseudoaneurysms of the hepatic arteries. J Am Coll Surg 1998; 186: 325-330.

6. Yamakado K, Nakatsuka A, Tanaka N, Takano K, Matsumura K, Takeda K: Transcatheter arterial embolization of ruptured pseudoaneurysms with coils and n-butyl cyanoacrylate. J Vasc Interv Radiol 2000; 11: 66-72.

7. Sato N, Yamaguchi K, Shimizu S, Morisaki T, Yokohata K, Chijiiwa K, Tanaka M: Coil embolization of bleeding visceral pseudoaneurysms following pancreatectomy: the importance of early angiography. Arch Surg 1998; 133: 1099-1102.

8. Cil BE: Transhepatic embolization of a recanalized congenital hepatic arterioportal fistula with NBCA and coils. Cardiovasc Intervent Radiol 2004; 27: 172-174.

9. Parildar M, Oran I, Memis A: Embolization of visceral pseudoaneurysms with platinum coils and N-butyl cyanoacrylate. Abdom Imaging 2003; 28: 36-40.

10. Murata S, Onozawa S, Nakazawa K, Akiba A, Mine T, Ueda T, Yasui D, Sugihara F, Kondoh Y, Kumita S: Endovascular embolization strategy for renal arteriovenous malformations. Acta Radiol 2014; 55: 71-77.

(Received, February 15, 2015)

(Accepted, July 7, 2015) 